Virginia Commonwealth University VCU Scholars Compass

2006

\title{
Determination of acceptor concentration in GaN from photoluminescence
}

\author{
Michael A. Reshchikov \\ Virginia Commonwealth University, mreshchi@vcu.edu
}

Follow this and additional works at: http://scholarscompass.vcu.edu/phys_pubs

Part of the Physics Commons

Reshchikov, M.A. Determination of acceptor concentration in GaN from photoluminescence. Applied Physics Letters, 88, 202104 (2006). Copyright (C) 2006 AIP Publishing LLC.

\section{Downloaded from}

http://scholarscompass.vcu.edu/phys_pubs/39

This Article is brought to you for free and open access by the Dept. of Physics at VCU Scholars Compass. It has been accepted for inclusion in Physics Publications by an authorized administrator of VCU Scholars Compass. For more information, please contact libcompass@vcu.edu. 


\title{
Determination of acceptor concentration in GaN from photoluminescence
}

\author{
M. A. Reshchikov ${ }^{\text {a) }}$ \\ Department of Physics, Virginia Commonwealth University, Richmond, Virginia 23284
}

(Received 5 January 2006; accepted 31 March 2006; published online 15 May 2006)

\begin{abstract}
The concentration of the acceptor responsible for the yellow luminescence (YL) band at about $2.2 \mathrm{eV}$ in $\mathrm{GaN}$ is determined from photoluminescence. The YL band intensity increases linearly with excitation power density and partially saturates above some critical value. The dependence is quantitatively described within a phenomenological model accounting for recombination statistics in GaN layer and saturation of acceptors with photogenerated holes. The incomplete saturation of the YL intensity at high excitation intensities is explained by gradual saturation of acceptors at different distances from the sample surface. The identity of deep and shallow acceptors in $\mathrm{GaN}$ is discussed. (C) 2006 American Institute of Physics. [DOI: 10.1063/1.2204835]
\end{abstract}

Gallium nitride has gained unprecedented attention due to its applications in emitters [green, blue, and ultraviolet light emitting diodes (LEDs) and laser diodes], optical detectors, and high-power amplifiers. However, even the bestquality GaN contains many point defects reducing the efficiency and lifetime of electronic and optical devices. To date, the majority of point defects in $\mathrm{GaN}$ remain unidentified and insufficiently studied. ${ }^{1}$ In particular, in undoped GaN grown by any technique a yellow luminescence (YL) band is commonly observed near $2.2 \mathrm{eV}$ that is attributed to transitions from the shallow donors or conduction band to an unidentified deep acceptor. ${ }^{1}$ The main candidate for the deep acceptor is the gallium vacancy-oxygen complex $\left(V_{\mathrm{Ga}} \mathrm{O}_{\mathrm{N}}\right)$ that is abundantly formed in $n$-type GaN during growth. ${ }^{2,3}$ In particular, the YL and green luminescence bands in high-quality freestanding $\mathrm{GaN}$ templates were assigned respectively to $2-/-$ and $-/ 0$ charge states of $V_{\mathrm{Ga}} \mathrm{O}_{\mathrm{N}} \cdot{ }^{4}$ However, there remains a controversy over the identification of the YL band in less pure material. ${ }^{1}$ In particular, Armitage et al. ${ }^{5}$ proposed that the carbon impurity in C-doped $\mathrm{GaN}$ and $V_{\mathrm{Ga}}$-related defects in undoped GaN cause YL bands very similar in shape and position.

The main difficulty in the identification of the YL band in $\mathrm{GaN}$ is associated with the common failure to determine the concentration of the related acceptor and compare it with the concentrations of the dominant impurities and vacancyrelated defects. The omnipresent method to determine the relative concentration of the acceptor from the relative intensity of the YL band in different samples is unacceptable since the photoluminescence (PL) intensity from a particular defect depends on many factors. ${ }^{6}$ Previously we proposed a method of how to determine the concentration of radiative acceptors in GaN from the analysis of the dependence of PL intensity on excitation intensity. ${ }^{6}$ However, at that time we could not explain satisfactorily the incomplete saturation of this dependence at excitation power densities corresponding to the saturation of the radiative acceptors with photogenerated holes. In this work we give an explanation of the incomplete saturation and determine the concentration of radiative acceptors in a GaN sample.

A $1.9-\mu \mathrm{m}$-thick unintentionally doped GaN layer was grown by metal organic chemical vapor deposition method

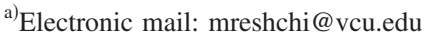

on $c$-plane sapphire substrate by EMCORE Corporation and studied by several research groups within the Wood-Witt Initiative "Defects in GaN" (Sample No. EM1256 for internal reference). Concentration of $\mathrm{Si}, \mathrm{C}$, and $\mathrm{O}$ in this sample has been estimated as about $3 \times 10^{16}, 4 \times 10^{16}$, and $5 \times 10^{16} \mathrm{~cm}^{-3}$, respectively, by the secondary ion mass spectrometry. ${ }^{7}$ Concentrations of free electrons at room temperature, $n_{0}$, shallow donors, $N_{D}$, and all compensating acceptors, $\Sigma N_{A}$, have been estimated as $2 \times 10^{16}, 6 \times 10^{16}$, and $4 \times 10^{16} \mathrm{~cm}^{-3}$, respectively, from the analysis of the temperature-dependent Hall effect. ${ }^{8}$ Steady-state PL was excited with unfocused $\mathrm{He}-\mathrm{Cd}$ laser $(55 \mathrm{~mW}, 325 \mathrm{~nm})$, dispersed by a $1200 \mathrm{rules} / \mathrm{mm}$ grating in a $0.3 \mathrm{~m}$ monochromator, and detected by a cooled photomultiplier tube. Calibrated neutral-density filters were used to attenuate the excitation power density $\left(P_{\text {exc }}\right)$ over the range of 1 $\times 10^{-5}-0.3 \mathrm{~W} / \mathrm{cm}^{2}$. Time-resolved PL was excited with a pulsed nitrogen laser $(337 \mathrm{~nm})$ and analyzed with an oscilloscope. A closed-cycle optical cryostat was used for temperatures between 10 and $320 \mathrm{~K}$.

PL spectra of the GaN sample at different temperatures are shown in Fig. 1. At $10 \mathrm{~K}$ the spectrum contains a sharp line at $3.484 \mathrm{eV}$ attributed to the annihilation of an exciton bound to a neutral shallow donor (DBE), followed by two LO phonon replicas, and a broad YL band with a maximum at $2.2 \mathrm{eV}$. With increasing temperature the DBE emission

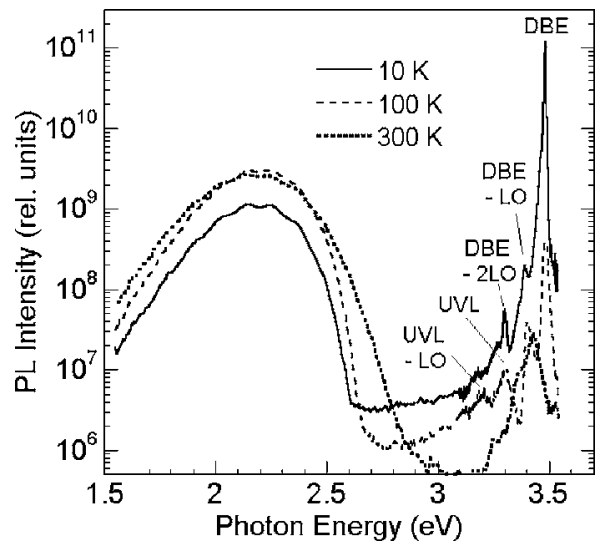

FIG. 1. PL spectrum from a GaN layer at different temperatures. The QE of different PL bands: $\eta_{\text {exciton }}=8 \%$ at $10 \mathrm{~K} ; \eta_{\mathrm{YL}}=2 \%$ at $10 \mathrm{~K}$ and $4 \%$ at $100-300 \mathrm{~K} ; \eta_{\mathrm{UVL}}=0.01 \%$ at $100 \mathrm{~K}$. 


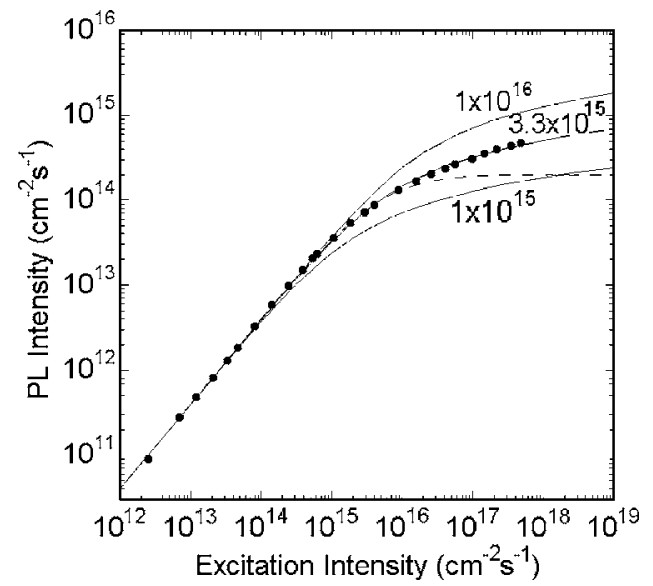

FIG. 2. Dependence of the YL intensity on excitation intensity at $300 \mathrm{~K}$. Points-experimental data. Solid lines—calculated by using Eq. (6) with the following parameters: $\alpha=10^{5} \mathrm{~cm}^{-1}, \tau=0.4 \mathrm{~ms}, \eta_{\mathrm{YL}}^{\text {exc }}=0.04, N_{\mathrm{YL}}=1 \times 10^{15}$, $3.3 \times 10^{15}$ and $10 \times 10^{16} \mathrm{~cm}^{-3}$.

quenches, giving way to the free exciton (FE) lines shifted by $\sim 6 \mathrm{meV}$ to higher energies. At temperatures close to $100 \mathrm{~K}$, when the FE emission significantly quenches, a very weak ultraviolet luminescence (UVL) band could be detected, representing a peak at $3.27 \mathrm{eV}$ followed by two $\mathrm{LO}$ phonon replicas (Fig. 1). At higher temperatures the UVL band quenches, and only the strong YL band and weak exciton band peaking at $3.428 \mathrm{eV}$ could be seen at room temperature (Fig. 1).

With increasing excitation intensity, $P_{0}$, up to $\sim 10^{15}$ photons $/ \mathrm{cm}^{2} \mathrm{~s}$, the YL intensity, $I^{\mathrm{YL}}$, increases linearly, and at higher excitation intensities it partially saturates (Fig. 2). By fitting the $I^{\mathrm{YL}}\left(P_{0}\right)$ dependence with the theoretical expression proposed in Ref. 6 ,

$$
I^{\mathrm{YL}}=P_{0}\left(\frac{P_{0} \tau_{\mathrm{YL}}}{L_{\mathrm{eff}} N_{\mathrm{YL}}}+\frac{1}{\eta_{\mathrm{YL}}}\right)^{-1},
$$

where $\tau_{\mathrm{YL}}$ and $\eta_{\mathrm{YL}}$ are the lifetime and quantum efficiency (QE) of the YL, $N_{\mathrm{YL}}$ is the concentration of acceptors responsible for the YL band, and $L_{\text {eff }}$ is "the effective thickness of the layer subject to excitation," "we obtain $N_{\mathrm{YL}} \approx 8$ $\times 10^{15} \mathrm{~cm}^{-3}$ by ignoring the discrepancy between the theoretical and experimental dependencies above $P_{0} \approx 1$ $\times 10^{16} \mathrm{~cm}^{-2} \mathrm{~s}^{-1}$ (Fig. 2). Note that for this evaluation the $\mathrm{QE}$ of the YL band $\left(\eta_{\mathrm{YL}}=0.04\right)$ has been estimated from the direct measurement of PL power with correction for the geometry of the PL registration optics. ${ }^{6}$ However, it is well known that the upward band bending of about $0.8 \mathrm{eV}$ exists near the surface due to the presence of negative charge at the surface of GaN. ${ }^{9}$ In the sample with the concentration of uncompensated donors of $2 \times 10^{16} \mathrm{~cm}^{-3}$ this band bending should create a $0.2-\mu \mathrm{m}$-thick depletion region near the surface from which the photogenerated carriers will be quickly swept out due to strong electric field, and the layer would be dead for PL. Therefore, the determined QE may be underestimated about ten times since the excitation intensity beyond the $0.2-\mu \mathrm{m}$-thick depletion region is reduced about ten times if the absorption coefficient $\alpha$ of $\mathrm{GaN}$ at $325 \mathrm{~nm}$ is about $10^{5} \mathrm{~cm}^{-1} .{ }^{10}$ Note also that the depletion region remains almost unchanged in the whole range of the excitation intensities used in this work. Indeed, under continuous illumination with our $\mathrm{He}-\mathrm{Cd}$ laser the band bending in $\mathrm{GaN}$ decreases by not more than $25 \%$ as compared to the dark. ${ }^{11}$

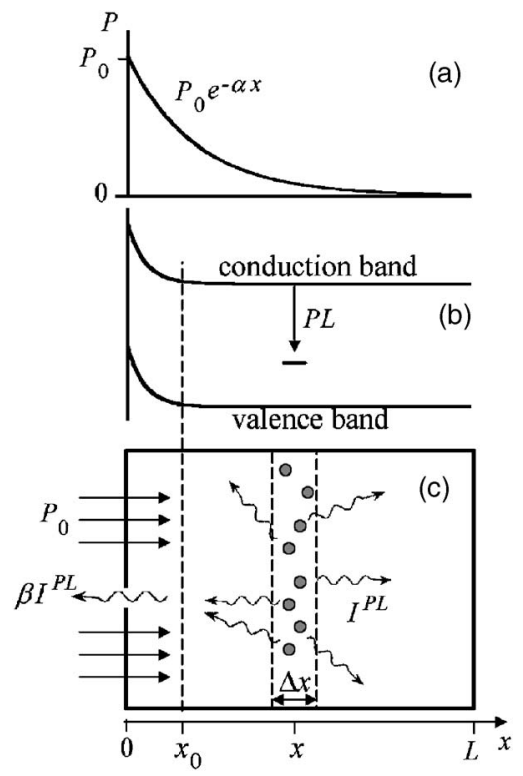

FIG. 3. Schematic of PL process. (a) Decrease of the excitation intensity inside GaN layer; (b) band diagram of the GaN layer having a depletion region between $x=0$ and $x=x_{0}$ due to upward band bending near the layer surface; (c) cross section of the GaN layer with selected thin layer $\Delta x$ where acceptors with the density $\Delta N=N_{i} \Delta x$ are shown as dots which emit PL in all directions. A fraction $\beta$ of the total PL intensity emitted from the layer is collected with a lens from the front side of the GaN layer.

Below we develop a phenomenological model for calculating the concentration of acceptors in $n$-type semiconductors accounting for the depletion region near the surface and gradual saturation of defects located at different depths of the layer with arbitrary thickness $L$ instead of using the effective thickness $L_{\text {eff. }}$.

Let us consider a GaN layer with thickness $L$ and depletion region width $x_{0}$ (Fig. 3). Laser intensity $P_{0}$ decreases inside the layer as $\exp (-\alpha x)$. At distance $x$, the following intensity will be absorbed within a thin layer $\Delta x$ :

$$
\Delta P(x)=\alpha P_{0} e^{-\alpha x} \Delta x .
$$

The number of acceptors related to the YL band in the thin layer of unit area is $\Delta N_{\mathrm{YL}}=N_{\mathrm{YL}} \Delta x$. Then the total intensity of the YL band emitted in the region from $x_{0}$ to $L$ in all directions is

$$
\begin{aligned}
I^{\mathrm{YL}} & =\int_{x_{0}}^{L} \frac{\alpha P_{0} e^{-\alpha x}}{\left(\alpha \tau_{\mathrm{YL}} P_{0} e^{-\alpha x} / N_{\mathrm{YL}}\right)+\left(1 / \eta_{\mathrm{YL}}\right)} d x \\
& =\frac{N_{\mathrm{YL}}}{\alpha \tau_{\mathrm{YL}}} \ln \left(\frac{P_{0}+P_{1}}{P_{0}+P_{2}}\right),
\end{aligned}
$$

where $\quad P_{1}=N_{\mathrm{YL}}\left(\alpha \tau_{\mathrm{YL}} \eta_{\mathrm{YL}} e^{-\alpha x_{0}}\right)^{-1} \quad$ and $\quad P_{2}$ $=N_{\mathrm{YL}}\left(\alpha \tau_{\mathrm{YL}} \eta_{\mathrm{YL}} e^{-\alpha L}\right)^{-1}$. At $P_{0}<P_{1}$ the $I^{\mathrm{YL}}\left(P_{0}\right)$ dependence is linear,

$$
I^{\mathrm{YL}}\left(P_{0}<P_{1}\right)=\eta_{\mathrm{YL}} e^{-\alpha x_{0}} P_{0}=\eta_{\mathrm{YL}}^{\mathrm{ext}} P_{0},
$$

where $\eta_{\mathrm{YL}}^{\mathrm{ext}}$ is the $\mathrm{QE}$ which can be found by direct measurements at low excitation intensity as $\eta_{\mathrm{YL}}^{\text {ext }}=I^{\mathrm{YL}} / P_{0}$. At $P_{0}>P_{2}$,

$$
I^{\mathrm{YL}}\left(P_{0}>P_{2}\right)=\frac{N_{i}}{\tau_{\mathrm{YL}}}\left(L-x_{0}\right) .
$$

Between $P_{1}$ and $P_{2}$ the $I^{\mathrm{YL}}\left(P_{0}\right)$ dependence is sublinear with gradually reducing slope. In typical $\mathrm{GaN}$ layers with thick- 
ness of more than $1 \mu \mathrm{m}$ the transition region between $P_{1}$ and $P_{2}$ extends on several orders of magnitude of $P_{0}$, and the complete saturation cannot be observed in the typical range of excitation intensities. Moreover, at very high excitation intensity the $I^{\mathrm{YL}}\left(P_{0}\right)$ dependence should become a square root dependence when the exciton emission becomes the dominant mechanism of recombination. ${ }^{6}$ Thus, for not very thin GaN layers $(L>1 \mu \mathrm{m})$ and not very high excitation power densities $\left(P_{\mathrm{exc}}<100 \mathrm{~W} / \mathrm{cm}^{2}\right)$, Eq. (3) may be simplified to

$$
I^{\mathrm{YL}}=\frac{N_{\mathrm{YL}}}{\alpha \tau_{\mathrm{YL}}} \ln \left(1+\frac{P_{0}}{P_{1}}\right)
$$

consisting of the linear region at $P_{0}<P_{1}$ and incomplete saturation region at $P_{0}>P_{1}$.

The experimental $I^{\mathrm{YL}}\left(P_{0}\right)$ dependence is fitted with Eq. (6) in Fig. 3. The lifetime of the YL at room temperature in the studied sample has been evaluated as $0.4 \mathrm{~ms}$ from the time-resolved PL study where a nearly exponential decay of the YL band intensity has been observed after pulse excitation. The value of $\eta_{\mathrm{YL}}^{\text {ext }}(0.04)$ was estimated from direct measurement of the QE by using a standard sample. ${ }^{6} N_{\mathrm{YL}}$ was the only fitting parameter, and the value of $N_{\mathrm{YL}}=3.3$ $\times 10^{15} \mathrm{~cm}^{-3}$ was found. Note that the proposed method for estimation of the concentration of the radiative defects is relatively accurate (see the fits for $N_{\mathrm{YL}}=10^{15}$ and $10^{16} \mathrm{~cm}^{-3}$ in Fig. 2) and does not require knowing the width of the depletion region. Note also that about twofold increase of the YL intensity with increasing temperature from 10 to $100 \mathrm{~K}$ (Fig. 1) favors the assumption that the $\mathrm{QE}$ of exciton emission is about $50 \%$ (at $10 \mathrm{~K}$ ) and the real internal QE of the $\mathrm{YL}\left(\eta_{\mathrm{YL}}\right)$ is close to $25 \%$ (at $300 \mathrm{~K}$ ). The difference between $\eta_{\mathrm{YL}}^{\mathrm{ext}}=4 \%$ and $\eta_{\mathrm{YL}}=25 \%$ is consistent with an assumption of a depletion region of about $0.1-0.2 \mu \mathrm{m}$ in the studied $\mathrm{GaN}$ sample.

Unfortunately we are still unable to identify the defect responsible for the YL band in GaN. The value of $N_{\mathrm{YL}}$ $=3.3 \times 10^{15} \mathrm{~cm}^{-3}$ is about an order of magnitude smaller than the total concentration of acceptors or major impurities, including $\mathrm{C}$ and $\mathrm{O}$, in the studied sample. Remarkably, we can estimate also the concentration of the shallow acceptor responsible for the UVL band in this sample, $N_{\mathrm{UVL}}$. Indeed, ${ }^{6}$

$$
N_{\mathrm{UVL}}=N_{\mathrm{YL}} \frac{I^{\mathrm{UVL}}}{I^{\mathrm{YL}}} \frac{C_{\mathrm{YL}}}{C_{\mathrm{UVL}}},
$$

where the hole capture coefficients for two acceptors can be taken as $C_{\mathrm{YL}}=3 \times 10^{-7}$ and $C_{\mathrm{UVL}}=1 \times 10^{-6} \mathrm{~cm}^{3} \mathrm{~s}^{-1}$ and the ratio of the integrated PL intensities between the YL and UVL bands at $100 \mathrm{~K}$ is 400 . This gives $N_{\mathrm{UVL}} \approx 3$ $\times 10^{12} \mathrm{~cm}^{-3}$. The result calls into question the possibility for $\mathrm{C}_{N}$ to be likely formed in GaN (Ref. 12) and be responsible for the UVL band. ${ }^{13,14}$

In conclusion, we have developed a new method for estimation of the radiative defects in semiconductors from the analysis of photoluminescence. Application of this method to unintentionally doped $n$-type GaN allowed us to estimate the concentration of deep acceptors responsible for the strong yellow luminescence band in this sample as 3.3 $\times 10^{15} \mathrm{~cm}^{-3}$. Concentration of the shallow acceptors in this sample is found to be 1000 times lower. The method is conceptually insensitive to the presence of depletion region near the surface of the semiconductor layer, which may significantly reduce the observed luminescence intensity.

The author thanks Dr. D. C. Look from Wright State University and Dr. S. Guo and Dr. D. Gotthold from EMCORE Corporation for providing GaN samples within the Wood-Witt initiative.

${ }^{1}$ M. A. Reshchikov and H. Morkoç, J. Appl. Phys. 97, 061301 (2005).

${ }^{2}$ J. Neugebauer and C. G. Van de Walle, Appl. Phys. Lett. 69, 503 (1996).

${ }^{3}$ T. Mattila and R. M. Nieminen, Phys. Rev. B 55, 9571 (1997).

${ }^{4}$ M. A. Reshchikov, H. Morkoç, S. S. Park, and K. Y. Lee, Appl. Phys. Lett. 81, 4970 (2002).

${ }^{5}$ R. Armitage, W. Hong, Q. Yang, H. Feick, J. Gebauer, E. R. Weber, S. Hautakangas, and K. Saarinen, Appl. Phys. Lett. 82, 3457 (2003).

${ }^{6}$ M. A. Reshchikov and R. Y. Korotkov, Phys. Rev. B 64, 115205 (2001).

${ }^{7}$ The SIMS data were provided by S-P. Guo and D.W. Gotthold (EMCORE).

${ }^{8}$ The electrical properties were provided by D.C. Look and Z.-C Fang (Wright State University)

${ }^{9}$ S. Sabuktagin, M. A. Reshchikov, D. K. Johnstone, and H. Morkoç, Mater. Res. Soc. Symp. Proc. 798, Y5.39 (2004).

${ }^{10}$ J. F. Muth, J. H. Lee, I. K. Shmagin, R. M. Kolbas, H. C. CaseyJr., B. P. Keller, U. K. Mishra, and S. P. DenBaars, Appl. Phys. Lett. 71, 2572 (1997).

${ }^{11}$ M. A. Reshchikov, S. Sabuktagin, D. K. Johnstone, and H. Morkoç, J. Appl. Phys. 96, 2556 (2004).

${ }^{12}$ A. F. Wright, J. Appl. Phys. 92, 2575 (2002).

${ }^{13}$ U. Birkle, M. Fehrer, V. Kirchner, S. Einfeldt, D. Hommel, S. Strauf, P. Michler, and J. Gutowski, MRS Internet J. Nitride Semicond. Res. 4S1, G5.6 (1999).

${ }^{14}$ E. R. Glaser, J. A. FreitasJr., B. V. Shanabrook, and D. D. Koleske, Phys. Rev. B 68, 195201 (2003). 